\title{
Photorefractive Crystals As Optical Devices, Elements, And Processors
}

Jeffrey O. White, Amnon Yariv

Jeffrey O. White, Amnon Yariv, "Photorefractive Crystals As Optical Devices, Elements, And Processors," Proc. SPIE 0464, Solid-State Optical Control Devices, (23 May 1984); doi: 10.1117/12.966219

SPIE Event: 1984 Los Angeles Technical Symposium, 1984, Los Angeles, United States 


\title{
Photorefractive Crystals as Optical Devices, Elements, and Processors
}

\author{
Jeffrey $O$. White and Amnon Yariv \\ Department of Applied Physics, California Institute of Technology \\ Pasadena, California 91125
}

\begin{abstract}
The physics of the photorefractive effect and the unique nonlinear optical properties of photorefractive crystals are described. A survey of the optical applications of the photorefractive effect follows. We include the use of photorefractive crystals as holographic storage elements, optical image amplifiers, phase conjugate mirrors, correction elements for intracavity laser distortions, phase conjugate windows for field transmission through inhomogeneous media, and coherent image processors.

Introduction

Since the discovery of the photorefractive effect in 1966, it has proven to be both a nuisance and an asset to the optics community. This paper concentrates on the latter area. Historically, the photorefractive effect was first considered to be optical "damage"; soon after, its potential as a holographic storage medium was realized. More recently, photorefractive crystals have been used as real-time Van der Lugt filters capable of replacing photographic film, as broadband phase conjugate mirrors, and as gain media with a mechanism quite different from stimulated emission. In the sections which follow, we describe these applications and draw comparisons between the photorefractive and conventional implementations.
\end{abstract}

The Photorefractive Effect

Study of the photorefractive effect began with the discovery of optical "damage" in $\mathrm{LiNbO}_{3}$ and $\mathrm{LiTaO}_{3}$ [1]. Ashkin et. al. noticed that the index of refraction of their crystals was changed by exposure to light, both coherent and incoherent. Visible and ultraviolet wavelengths produced the effect, but not infrared. If the illumination was nonuniform, the optical homogeneity of the crystals suffered and it was feared that such sensitivity would make $\mathrm{LiNbO}_{3}$ less useful as a nonlinear medium. The damage remained when the light was removed, yet the effect was reversible, in contrast to the catastrophic damage that occurs at much higher intensities.

The utility of $\mathrm{LiNbO}_{3}$ for frequency doubling and waveguiding was threatened, and research has continued on how to reduce the susceptibility to optical damage [2]. On the other hand, optical damage represented a new type of nonlinearity which was useful in its own right. There is a corresponding effort to enhance the photorefractive effect by doping [3], and chemical reduction [4,5]. Both efforts have been furthered by studies of the photorefractive mechanism through photoelectron spectroscopy [6], fluorescence spectroscopy [7], Mossbauer and electron paramagnetic resonance methods [8].

Basic Mechanism

In the broadest sense, photorefractive materials could include any material in which light could produce an index change. The term photorefractive effect is used most commonly today with reference to the combination of a photoinduced space charge field and the electro-optic effect. Only in photoconducting electrical insulators has this been observed to date, and amongst them only in ferroelectrics, e.g., $\mathrm{LiNbO}_{3}, \mathrm{LiTaO}_{3}, \mathrm{BaTiO}_{3}$, and $\mathrm{KNbO}_{3}$, and the paraelectric sillenites, bismuth silicon oxide $\left(\mathrm{Bi}_{12} \mathrm{SiO}_{20}, \mathrm{BSO}\right)$ and bismuth germanium oxide $\left(\mathrm{Bi}_{12} \mathrm{GeO}_{20}, \mathrm{BGO}\right)$. Another characteristic which photorefractive materials have in common are localized charge states, or traps, within the energy gap.

The first explanation of the photorefractive effect was due to Chen and Amodei [9,10]. Their hypothesis was that trapped charges migrated under the influence of the light. The migration consists of optical excitation from the traps into the conduction band, diffusion and drift, followed by a return to the traps via collisional recombination. The excitation is preferential in the regions of high intensity, and the trapping is at random, so there is a net migration of charge from the intensity maxima to the minima, leaving behind positively ionized donors, i.e. trapped holes. The electric field associated with the space charge density creates an index variation via the electrooptic effect. The index variation has a spatial variation similar to that of the light exposing the crystal (see Fig. 1).

When the current in the conduction band is due to diffusion only, the index grating is $\pm \pi / 2$ out of phase with the interference pattern, depending on the sign of the effective electrooptic coefficient or the sign of the space charge field with respect to the positive $\mathrm{c}$ axis of the crystal. A uniform dc external field can increase the amplitude of the variation of the space charge field, and change the phase shift.

Because the crystal is an insulator in the dark, when the light is turned off, all the electrons must leave the conduction band. The space charge is lost if they all return to ionized sites of the original type, i.e. if all the electrons recombine with trapped holes. Space charge storage is only possible if a population of holes in the donor sites exists in the dark: nocturnal holes. This requires the presence of a second alternative type of site for the electrons to reside. 


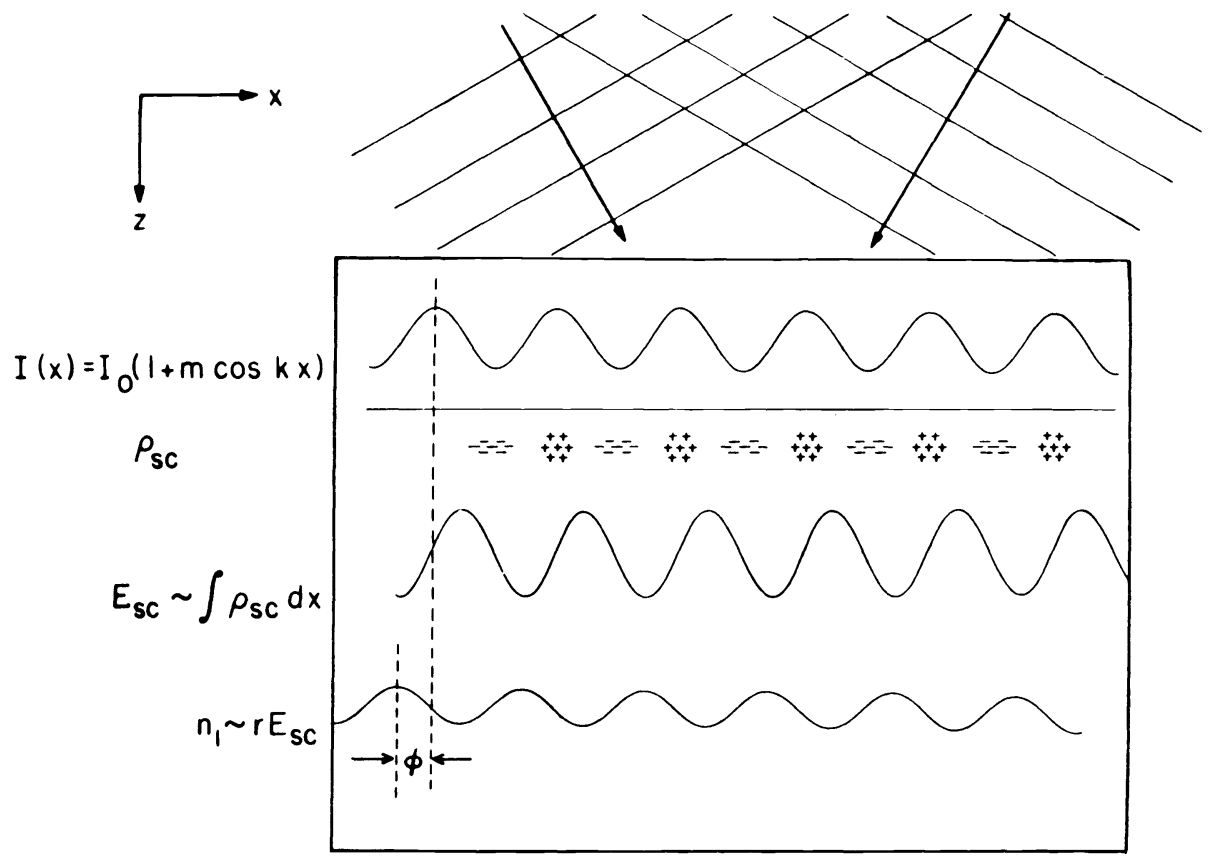

Figure 1. The photorefractive mechanism.

The photorefractive effect has been explored in many ways [11]. Theoretical work has made use of charge transport equations coupled to Maxwell's equations [12], and statistical mechanics [13]. A basic experiment is to write a grating with two beams, and monitor its diffraction efficiency with a third, probe beam. If the energy of the probe beam photons is insufficient to excite free electrons, then it will perform a nondestructive readout. The major observation has been that the amplitude of the space charge field, $E_{1}$, is proportional to the modulation index, $\mathrm{m}$, of the intensity variation which drives it [14], hence the diffraction efficiency exhibits a quadratic dependence on $\mathrm{m}$. The steady state grating amplitude has been found to increase with absolute writing intensity until a gradual saturation. The saturation occurs at $600 \mu \mathrm{W} / \mathrm{cm}^{2}$ in BSO [15], and over $100 \mathrm{~W} / \mathrm{cm}^{2}$ in heavily doped $\mathrm{KNbO}_{3}[4]$.

\section{Optical Storage Devices}

The light induced charge migration within photorefractive crystals is most efficient for illumination with rapid spatial variation, so the photorefractive effect is well suited for storing speckle patterns, or for storing slowly varying intensities which have modulated a spatial carrier frequency [16].

Chen, LaMacchia and Fraser were the first to realize the opportunities that the optical damage presented by demonstrating that holograms could be written, stored and erased in $\mathrm{LiNbO}_{3}$ [17]. In conventional holography the interference pattern between an object beam and a reference beam falls on photographic film, recording the amplitude and phase of the object beam. The spatial variation of intensity is mapped into a spatial variation of silver concentration ( or absorption constant ) when the film is developed. In real-time holography, the interference pattern fills the crystal, and changes its index as the exposure takes place. Erasure is performed by illuminating with uniform light which effects a uniform redistribution of the trapped charge.

Interest is optical memories is motivated by potentially very high (diffraction limited) storage densities of $10^{8} \mathrm{bits} / \mathrm{cm}^{2}$. In holographic optical memories, the information is distributed throughout the hologram. Such spatial redundance can be advantageous because, in the event of loss of part of the hologram, the decrease in SNR is distributed as well. Volume holograms have the additional characteristic of angular selectivity, which allows separate holograms to be stored in the same volume. Photorefractive crystals appear to be well suited to this application because their sensitivity $\left(300 \mu \mathrm{J} / \mathrm{cm}^{2}\right.$ yields a $1 \%$ diffraction efficiency hologram in BSO [15]) is comparable to photographic film, they are self-developing, and erasable. The best insulators have long dark storage times (30-100 hours in BSO) which are promising for optical memories except that erasure occurs during readout.

A method has been discovered whereby the holograms can be fixed if they are recorded at about $200^{\circ} \mathrm{C}$ and then cooled to room temperature [18]. Using this technique, 500 holograms were stored in a $1 \mathrm{~cm}$. thick crystal of $\mathrm{LiNbO}_{3}: \mathrm{Fe}$, a density of $10^{10} \mathrm{bits} / \mathrm{cm}^{3}$. The diffraction efficiency of each hologram was $\geq 2.5 \%$, and the estimated lifetime of $10^{5}$ years is suitable for archival storage. 
Current research in holographic storage includes quantitatively evaluating the performance of prototype systems which use the photorefractive effect [19,20], interfacing to the incoherent world with light valves [21], and determining the effect of readout with red light [22]. As might be expected in view of their holographic applications, photorefractive crystal's have been used to store interference patterns for use in time-averaged and double-exposure interferometry as well [23,24].

Photorefractive crystals are most commonly used today in Pockel's Readout Optical Modulators (PROM's), which can be used to store two-dimensional images, process them, and convert incoherent to coherent images. PROM's make use of the same properties of electrical insulation, photoconduction and linear electrooptic effect, although the charge migration is intended to be strictly in accordance with an external field, rather than an intensity gradient. Recent work has sought to make use of the diffusion mechanism, in conjunction with an external field, to construct a simpler, more passive, high resolution image converter [25-27].

\section{Coherent Optical Amplifiers}

Six years after the discovery of the photorefractive effect, an energy exchange was observed between two beams used to write a hologram in $\mathrm{LiNbO}_{3}$ [28]. This exchange can be qualitatively understood through a coupled wave theory of Bragg diffraction within fixed, thick, gratings [29]; and was originally explained in such terms even though the exchange was observed within a dynamic medium.

The index of refraction and optical field within a fixed, thick grating are taken to be

$$
n=n_{0}+\frac{n_{1}}{2} e^{-i(z \cdot r+\varphi)}+\frac{n_{1}}{2} e^{i(z \cdot r+\varphi)} \quad E(r, t)-A_{1}(z) e^{i(\omega t-\mathbf{k} \cdot r)}+A_{2}(z) e^{i(\omega t-\mathbf{k} \cdot r)}
$$

where the slowly varying complex amplitude, or envelope, of each wave varies with distance inside the grating due to the coupling between the two waves. Both are assumed to be at the Bragg angle of incidence, $\theta$, such that $\mathbf{k}_{1}-\mathbf{k}_{2}-\mathbf{g}$. The coupled wave equations are derived from the scalar wave equation by neglecting the second order spatial derivative of the envelope in comparison to the optical wavevector times the first order derivative. One can solve for the amplitude for a wave to be transmitted or diffracted by the grating.

$$
t=\cos \left(n_{1} d / \lambda \cos \theta\right) \quad r=i \sin \left(n_{1} d / \lambda \cos \theta\right)
$$

where $d$ is the thickness of the grating. The $i$ represents the $\pi / 2$ phase shift upon reflection from a periodic index variation.

When two waves are incident upon the grating, the transmitted part of beam 1 interferes with the diffracted part of beam 2, and vice versa. Fig. 2a depicts a case where both incident beams have unit amplitude.
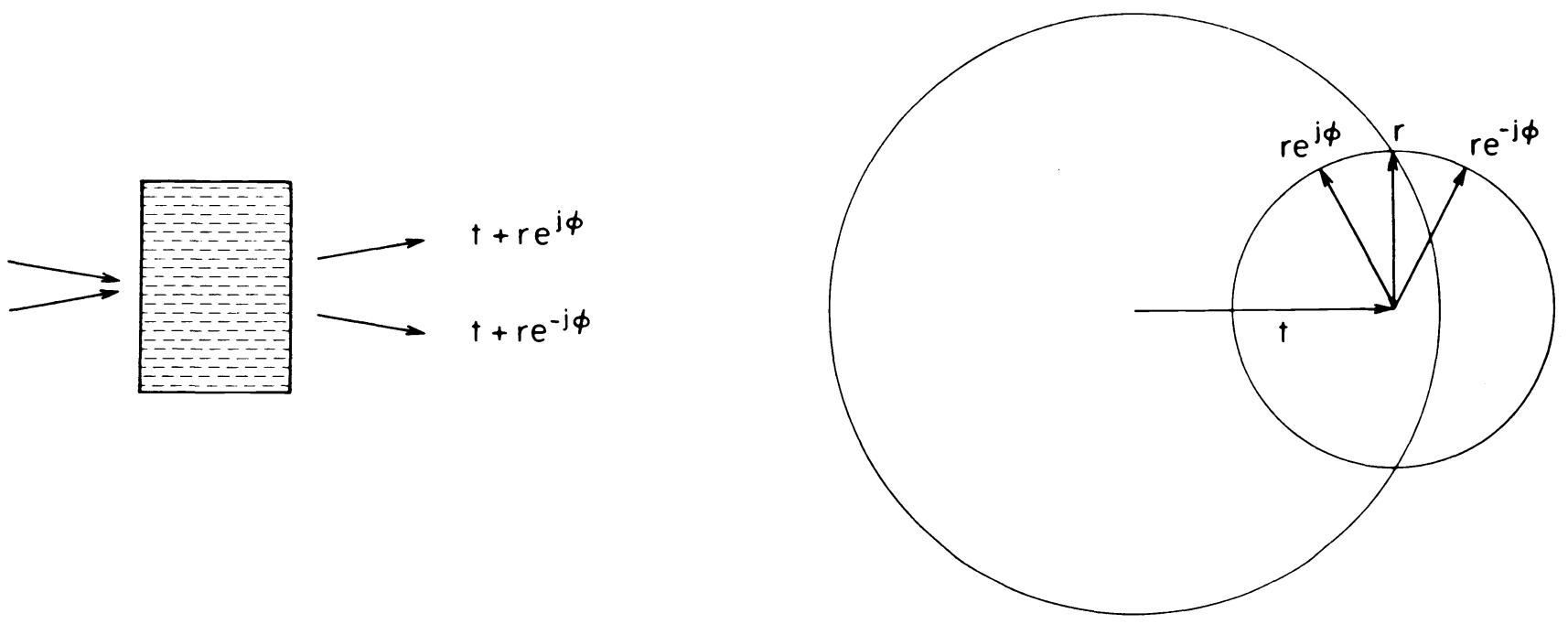

Figure 2. Energy exchange in a fixed grating. a) The incident beams may have equal amplitudes. b) The amplitudes of the output beams may be computed geometrically.

As the grating is translated in the direction of $\mathrm{g}$, the phase $\varphi$, of the fixed grating relative to the interference pattern at the input face, enters the problem. The transmitted parts of both beams are unaffected because they see the average index, but the reflected parts are affected oppositely, i.e., the phase of one beam is advanced, the other is delayed. The amplitudes of the upper and lower transmitted beams will be $t+r e^{j \varphi}$, and $t+r e^{-j \phi}$, respectively. The sum of $t$ and $r$ lies on the unit circle in the case of a lossless grating. Figure $2 b$ shows that the maximum energy exchange occurs for a phase angle of $\pi / 2$. This is exactly the 
intrinsic phase angle that naturally occurs, throughout the volume of a photorefractive crystal, when the current in the conduction band is due to diffusion only.

Steady state energy transfer in nonlocal media

In the coupled wave theory of dynamic gratings [30], the response of the index of refraction of the medium is chosen to be proportional to the modulation index of the incident intensity variation, $2 A_{1} A_{2}^{*} /\left(I_{1}+I_{2}\right)$, where $I_{i}=\left|A_{i}\right|^{2}$.

$$
n=n_{0}+n_{1} e^{-i \varphi} \frac{A_{1} A_{2}^{*}}{I_{1}+I_{2}} e^{-i\left(\mathbf{k}_{1}-\mathbf{k}_{2}\right) \cdot r}+n_{1} e^{i \varphi} \frac{A_{1}^{i} A_{2}}{I_{1}+I_{2}} e^{i\left(\mathbf{k}_{1}-\mathbf{k}_{2}\right) \cdot r}
$$

The phase factor, $\varphi$, represents the intrinsic spatial phase shift between the interference pattern and the induced index grating. Separating the complex amplitudes into a magnitude and a phase, one can show that at each point in space, the intensities are coupled through the sine of the phase shift, and the phases are coupled through the cosine

$$
\begin{array}{ll}
\cos \theta \frac{d I_{1}}{d z}=-\alpha I_{1}-\frac{2 \pi n_{1}}{\lambda} \sin \varphi \frac{I_{1} I_{2}}{I_{+}} & \cos \theta \frac{d \varphi_{1}}{d z}=\frac{\pi n_{1}}{\lambda} \cos \varphi \frac{I_{2}}{I_{+}} \\
\cos \theta \frac{d I_{2}}{d z}=-\alpha I_{2}+\frac{2 \pi n_{1}}{\lambda} \sin \varphi \frac{I_{1} I_{2}}{I_{+}} & \cos \theta \frac{d \varphi_{2}}{d z}=\frac{\pi n_{1}}{\lambda} \cos \varphi \frac{I_{1}}{I_{+}}
\end{array}
$$

where $I_{+}-I_{1}+I_{2}$, and the linear absorption, which is crucial to the photorefractive effect, is denoted by $\alpha$. For a transmission geometry, such as that shown in Fig. 3, the solution is given by

$$
\begin{array}{lll}
I_{2} & I_{1}=I_{1}(0) e^{-a r} \frac{I_{1}(0)+I_{2}(0)}{I_{1}(0)+I_{2}(0) e^{2 \Gamma r}} \\
& I_{2}=I_{2}(0) e^{-a r} \frac{I_{1}(0)+I_{2}(0)}{I_{1}(0) e^{-2 \Gamma r}+I_{2}(0)}
\end{array}
$$

Figure 3. Two-wave mixing in the transmission geometry.

where $\Gamma=\frac{\pi \mathrm{n}_{1}}{\lambda} \sin \varphi$ and $\mathrm{r}$ is the path length of each beam, i.e. $r=\mathrm{z} / \cos \theta$.

At the exit face of the crystal, for large, positive $\Gamma$, the sum of the intensities of both beams appears in $\mathrm{I}_{2}$, aside from the linear absorption. This represents coherent optical gain for beam 2, and loss for beam 1. Input/output curves for such an amplifier, in the (artificial) case of no absorption, are shown in Fig. 4. This type of energy transfer has been used to coherently amplify nonplanar object waves which carry information. A $15 \mathrm{nW} / \mathrm{cm}^{2}$ object beam has been amplified by a factor of 4000 by an amplifier consisting of a $2.4 \mathrm{~mm}$ thick crystal of $\mathrm{BaTiO}_{3}$ pumped by $150 \mathrm{~mW}$ of $514.5 \mathrm{~nm}$ light from an argon ion laser [31]. The absence of excited states in the amplifier eliminates the noise due to spontaneous emission. At present, the slow response time of photorefractive crystals limits their use to coupling two beams supplied by the same laser. Using a portion of the laser output to pump an amplifier of this type would appear to be most useful when the object could be damaged by directing all the laser power into the object wave.

Transient energy transfer in local media

In media with local response, i.e., $\varphi-0$, one would not expect to see steady state energy transfer, but a transient energy transfer can be observed [32]. It is a consequence of the inertia or finite response time of the photorefractive effect. The grating begins to form initially in phase with the interference pattern. This couples the phases of the two beams and shifts the interference pattern so that the two are brought out of coincidence within the volume of the crystal (Fig. 5a). Before the grating can catch up with the interference pattern, the exchange takes place. In steady state, the two are in coincidence and there is no energy exchange (Fig. 5b).

Oscillation in two-wave mixing

Ten years after the energy exchange was observed, it was combined with mirror feedback to produce unidirectional oscillation in a ring resonator [33]. The gain medium was $\mathrm{BaTiO}_{3}$, pumped by a HeNe laser operating at $632.8 \mathrm{~nm}$. Here, initially, no grating is present because only one beam, the pump, is supplied (Fig. 6). Using boundary conditions appropriate to a ring resonator, one can solve for the threshold coupling strength $2 \Gamma l \geqslant a l-\log (1-\mathrm{S})$ where $l-\mathrm{d} / \cos \theta$, and $\mathrm{S}$ represents the losses due to mirror transmission. The threshold is independent of pump intensity, a consequence of the index variation being driven by intensity modulation, not absolute intensity. This conclusion was checked experimentally. Even with a pump intensity of 15 $\mathrm{mW} / \mathrm{cm}^{2}$, an oscillation could build up inside the cavity. At that particular intensity, the time to reach steady state is eight minutes. It is not known whether the oscillation builds up from scattered light or from the zero point energy in the cavity, but it is known that the amplification is not via stimulated emission so "laser" is not an appropriate name for such an oscillator. The oscillation is unidirectional, indicating that the $\Gamma$ for the counterclockwise beam is below the threshold. 


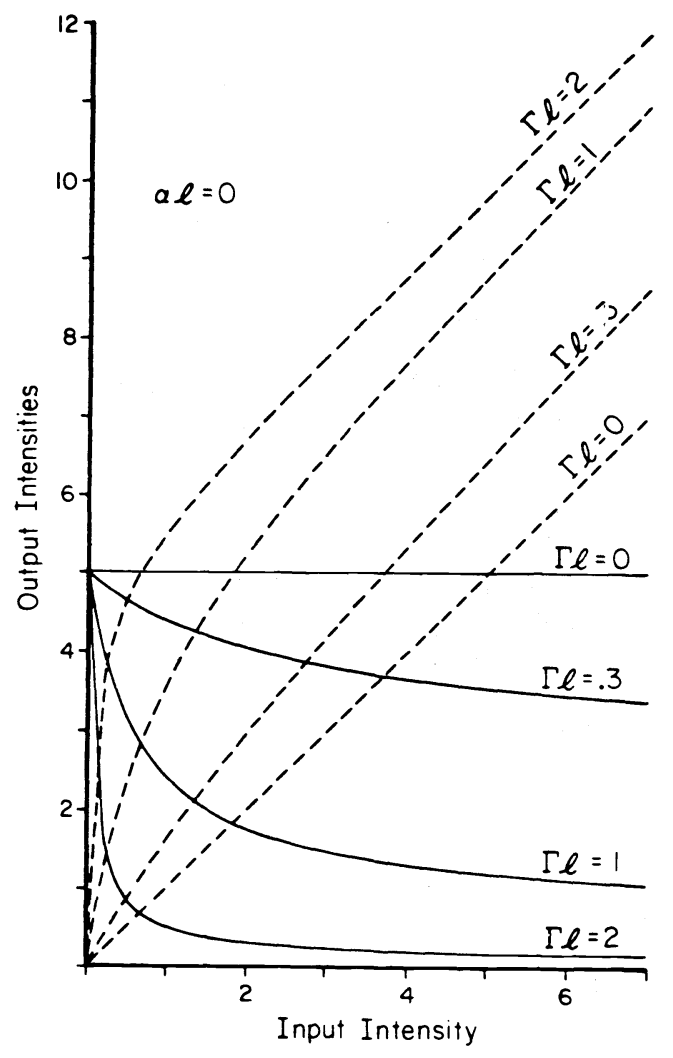

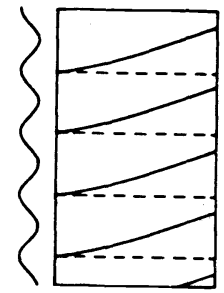

5.

a)

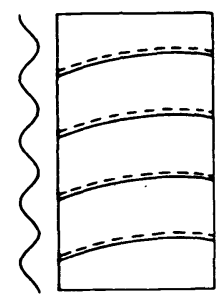

b)

4.

6.

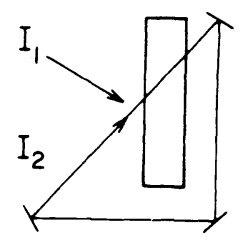

Figure 4. Input/output curves for a two-wave mixing amplifier. The output intensities of both beams are plotted vs. the input intensity of the signal beam. The broken line is the output intensity of the signal beam and the solid line is the output intensity of the pump beam. The input intensity of the pump beam is fixed at 5 .

Figure 5. a) Transient energy exchange due to inertia in media with local response. Solid lines are the interference pattern, broken lines are the grating. b) In steady state the grating and interference pattern are coincident, and there is no energy exchange.

Figure 6. Unidirectional ring resonator with a photorefractive crystal providing the gain and directionality.

It is possible to excite a pure higher order transverse mode with a gain medium of sufficient optical quality, as shown in Fig. 7 [31]. The slow frequency response of photorefractive crystals at practical intensities, compared to the frequency difference, between longitudinal modes in a cavity of practical length, will make it difficult to observe an oscillation with longitudinal modes at frequencies differing significantly from those in the pump wave.

\section{Phase Conjugate Mirrors}

Photorefractive crystals have recently been used to perform phase conjugation [14]. The phase conjugate replica of a monochromatic optical field is a field, of the same frequency, whose wavefronts take the same shape throughout space, but propagate in the opposite direction at every point. A wave travelling essentially in the positive $\mathrm{z}$ direction is denoted by

$$
E_{1}(r, t)=A_{1}(r) e^{i(\omega t-k z)}+A_{i}^{i}(r) e^{-i(\omega t-k z)}
$$

where the complex amplitude $A_{1}(r)$ can describe any spatial amplitude or phase information impressed upon $E_{1}$. Mathematically, its phase conjugate is obtained by complex conjugating the spatial part

$$
E_{P C}(r, t)-A_{i}^{*}(r) e^{i(\omega t+k z)}+A_{1}(r) e^{-i(\omega t+k z)}
$$

or changing $\mathrm{t} \rightarrow-\mathrm{t}$, hence the identification of phase conjugation with "time reversal". A phase conjugate mirror (PCM) is a device which generates the phase conjugate of an incident wavefront. In an ideal two mirror laser cavity, the counterpropagating fields are phase conjugates of each other, so both mirrors are performing wavefront reversal. However, a true PCM will generate the phase conjugate replica of an incident wavefront possessing arbitrary spatial variation of amplitude, phase, and 

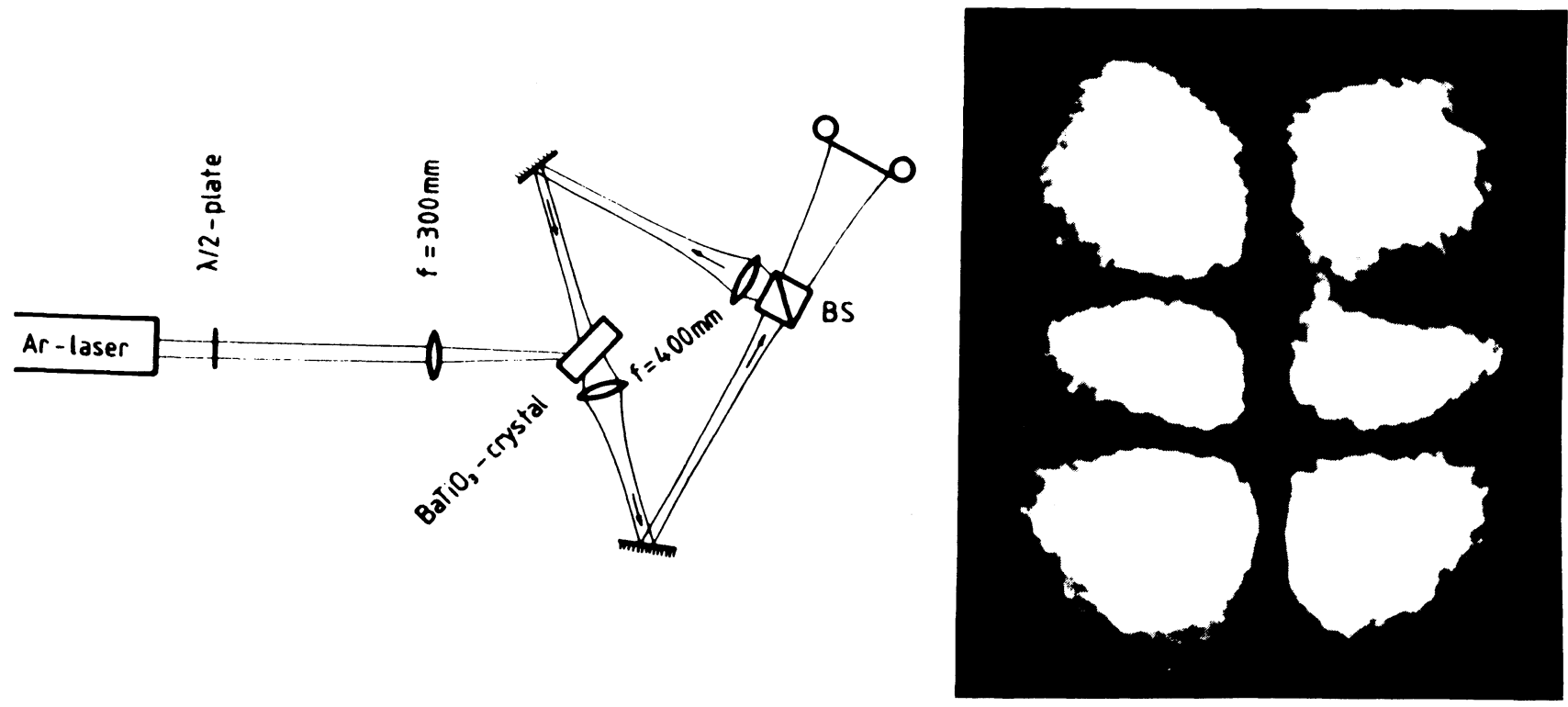

Figure 7. Higher order transverse mode inside a ring resonator with a photorefractive gain element [31].

\section{polarization.}

Much of the interest in phase conjugate waves is due to their distortion correcting capability. Figure 8 illustrates a double pass distortion correction scenario where an undistorted wave (1) passes through a lossless region of nonuniform index of refraction, the distorted wave (2) is incident upon a PCM, and the phase conjugate wave (3) returns through the distortion and emerges aberration free (4). The distortion in the figure could represent modal dispersion in a fiber, atmospheric turbulence, thermal blooming, imperfect optics, etc.
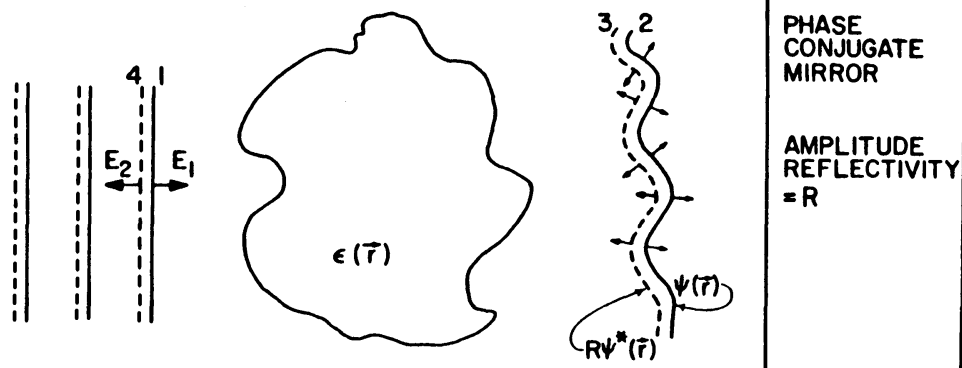

Figure 8. Typical geometry depicting the ability of phase conjugation to correct for a general spatially dependent phase aberration [34].

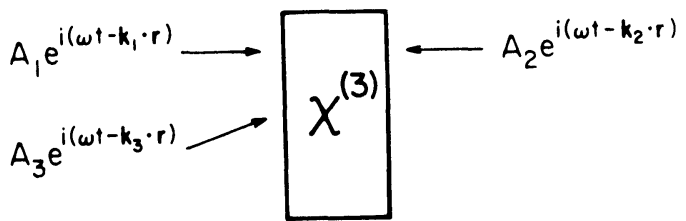

$$
\begin{aligned}
P_{N L} & =\chi^{(3)} A_{1} A_{2} A_{3}^{*} e^{i(\omega+\omega-\omega) t} e^{-i\left(k_{1}+k_{2}-k_{3}\right) \cdot r} \\
& =\chi^{(3)} A_{1} A_{2} A_{3}^{*} e^{i\left(\omega t+k_{3} \cdot r\right)}
\end{aligned}
$$

Figure 9. The four-wave mixing implementation of a phase conjugate mirror.

Phase conjugate mirrors via linear optics

The first realizations of phase conjugate mirrors were via Coherent Optical Adaptive Techniques [35]. These systems have achieved compensation for aberrated wavefronts with the use of electrooptic devices, acoustic devices, or deformable "rubber" mirrors. The latter are thin metallic reflectors supported by arrays of piezoelectric actuators. The actuators are driven by wavefront error sensors. 
Conventional holographic techniques can be used for imaging through stationary distortions [36]. One need only 1) create a hologram of the distorted object wave and 2) illuminate it with a reconstructing wave counterpropagating to the reference wave. This generates a phase conjugate wave which will retrace the path of the object wave through the distortion and converge to a real image.

Phase conjugate mirrors via nonlinear optics

Stimulated backscattering (Brillouin or Raman) can generate phase conjugate replicas of incident waves with sufficient intensity [37,38]. A forward going optical wave in a nonlinear medium, e.g. $\mathrm{CS}_{2}$, stimulates a forward going phonon wave, which possesses the same wavefront, and perturbs the index of refraction of the medium. Part of the incident optical wave is retroreflected by the index perturbation, and this constitutes a Stokes shifted phase conjugate wave.

The study of all-optical parametric processes in nonlinear media led directly to the current activity in the field. Several review articles have discussed both theoretical and experimental aspects of phase conjugation, its applications, and the nonlinear mechanisms and media used to date [39,41]. The most common implementation of a phase conjugate mirror consists of a medium possessing a third order susceptibility pumped by two counter-propagating optical fields, $A_{1}$ and $A_{2}$, with frequency $\omega$ (Figure 9). An incident, signal wave, $A_{3}$, combines with the pump waves to induce a third order nonlinear polarization in the medium. The polarization radiates a fourth, phase conjugate wave at $\omega$, hence the name degenerate four-wave mixing (D4WM). Each plane wave component of $\mathrm{A}_{3}$ induces a corresponding component of the phase conjugate wave travelling in exactly the opposite direction. Four-wave mixing has become the most important nonlinear optical technique for generating phase conjugate waves because the interaction is automatically phase matched for all angular components of the incident wave, a third order susceptibility is not forbidden in any material on symmetry grounds, and an amplified reflection is possible [42].

Nonlinear optical implementations of PCM's have several advantages:

i. The response time is limited only by the nonlinear medium itself. The processing of different parts of the incident wave takes place in parallel.

ii. The resolution in the near field is limited by the density of the atomic or molecular species participating in the interaction, or the wavelength of the incident light.

iii. The possibility of gain exists as part of the phase conjugation process itself.

iv. Wavefront error sensors are not necessary.

and several disadvantages:

i. Many types of nonlinear media are not available in large sizes of good optical quality. The size of a PCM will limit its resolution in the far field of a distortion.

ii. In $4 \mathrm{WM}$, the pump beams have to be coherent with respect to the signal wave, to a degree determined by the response time of the medium.

Many types of nonlinear media have been used as the mixing medium inluding two-level systems, dyes, semiconductors, radiatively cooled vapors, plasmas, liquid crystals, and aerosols. In view of the formal analogy between holography and 4WM [43], it is not surprising that photorefractive media have been widely used for both. The photorefractive media are particularly suitable for use with low power continuous wave lasers because of their extraordinary sensitivity. Photorefractive PCM's differ from those constructed with conventional nonlinear media [44], for example, a crystal must lack inversion symmetry to possess the linear electrooptic effect. One advantage is that photorefractive nonlinearities are as large as those of resonant media, but photorefractive crystals are broadband, i.e., they respond to light throughout the visible spectrum. They are not broadband in the sense of responding to rapid temporal variations, e.g., the fringe pattern formed by two optical frequencies which differ by a MHz.

Spectral response

The slow response time of the photorefractive effect is the basis for a narrow band filter [45]. The filter consists of a photorefractive crystal pumped by two waves whose frequency determines the center frequency of the band pass region. The only signal waves which are coupled to the pump waves are those with frequencies which can form a stationary or slowly moving grating with the pump wave. Since the response time is inversely proportional to the intensity, the pass band increases as the pump intensity increases.

Self-pumped phase conjugate mirrors

One hindrance to the practical utilization of PCM's has been the need to supply pump waves which are coherent with respect to the signal wave. Several schemes have been developed to remove this requirement. In the configuration of Fig. 10, the crystal is placed within a Fabry-Perot resonator. Gain is provided by the single signal wave incident upon the photorefrac- 
tive crystal, and the oscillation which builds up is shown by the dotten line. To the extent that the oscillating beams are phase conjugates of each other, they will generate the phase conjugate of the signal wave via D4WM. This configuration is called a passive phase conjugate mirror (PPCM) because the counterpropagating pump beams do not have to be supplied externally $[33,46]$.

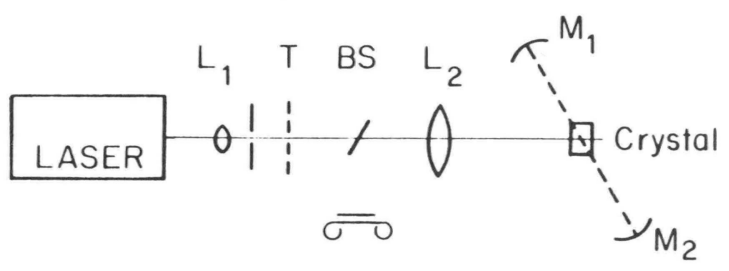

Figure 10. Imaging with a self-pumped phase conjugate mirror. $\mathrm{L}_{2}$ is not an imaging lens, it only serves to direct more of the object wave, which originates at transparency $T$, onto the crystal. BS is a beamsplitter which directs the phase conjugate wave to the image plane.

Reflectivities greater than one are impossible in steady state, but reflectivities approaching one are theoretically possible, and $30 \%$ has been observed. The alignment of the Fabry-Perot cavity is critical, but the alignment of the cavity axis relative to the incident wave is not. An acceptance angle of $20^{\circ}$ on either side of the crystal has been measured.

Laser with dynamic holographic intracavity distortion correction

Distortions in lasers commonly arise because of defects in laser rods, turbulence in gas discharges, imperfect optics, thermal effects, and nonlinear effects. Compensation has been achieved using the PPCM described in the last section to replace one of the normal mirrors in a laser [47]. A piece of etched glass was used to simulate a distortion in a commencial argon ion laser. The distortion correcting capability is apparent from the pictures of the output shown in Fig. 11.

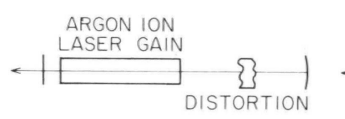

(a)

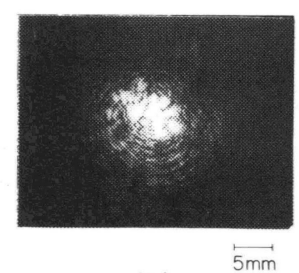

(b)

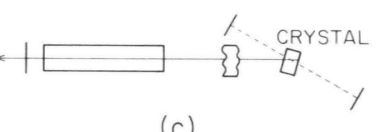

(c)

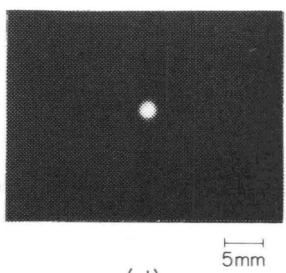

(d)

Figure 11. Laser with dynamic holographic intracavity distortion correction capability.

Inserting the etched glass into the normal laser cavity (Fig. 11a) destroyed the mode (Fig. 11b) and lowered the output power from $2 \mathrm{~W}$ to $1 \mathrm{~mW}$. Replacing the normal mirror with a PPCM (Fig. 11c) restored the mode shape (Fig. 11d) and boosted the power output back to $0.5 \mathrm{~W}$.

Several other self-pumped lasers have been developed, all of which make use of the photorefractive effect. One version makes use of total internal reflection from crystal faces to replace the external Fabry-Perot cavity [48]. This type has been used as the end mirror on a dye laser [49]. Another version has been used as the end mirror on a Cu vapor laser, wherein the spontaneous emission from the laser is sufficient to initiate operation [50].

\section{Information Transmission}

A very common problem is the need to transmit optical information through a distorting medium. A beam propagating through the atmosphere can be distorted by turbulence, or, if the beam is intense enough, it will also encounter, or create, nonlinear distortions such as thermal blooming. Aberration compensation can be performed before a distorted image is received (predetection) or after (postdetection). The double pass distortion correction geometry (Figure 8) is of no use because the corrected image is obtained on the same side of the distortion as the object.

With the aid of a co-propagating reference wave

A predectection scheme using conventional holography requires the presence of a reference wave originating on the same side of the distortion as the object wave [51]. If both waves propagate through essentially the same path, on the receiving side the distortions can be removed in the recording process by mixing (interfering) the two distorted waves. If the mixing were done via dynamic holography, one could transmit information in real-time through a turbulent distortion. The reconstruction 
takes place when the hologram is illuminated with an undistorted reference.

With the aid of a counter-propagating reference wave

For situations where the object and reference are located on opposite sides of an aberrating path, a new scheme for one way field transmission has been proposed and demonstrated [52,53]. The technique is limited to purely phase distortions characterized by a multiplicative complex amplitude to be transmitted $t=\mathrm{e}^{\mathrm{i} \phi(x, y)}$, where $\varphi$ can be a random function of the transverse coordinates. If the distortion and its conjugate, $t^{*}=e^{-i \phi(x, y)}$, were in contact, the combination of the two would be homogeneous, i.e. $\mathrm{tt}^{*}=1$, independent of $\mathrm{x}$ and $\mathrm{y}$. The technique consists of creating the conjugate distortion in real-time within a nonlinear medium and imaging the nonlinear medium and the distortion together with a lens (Fig. 12).

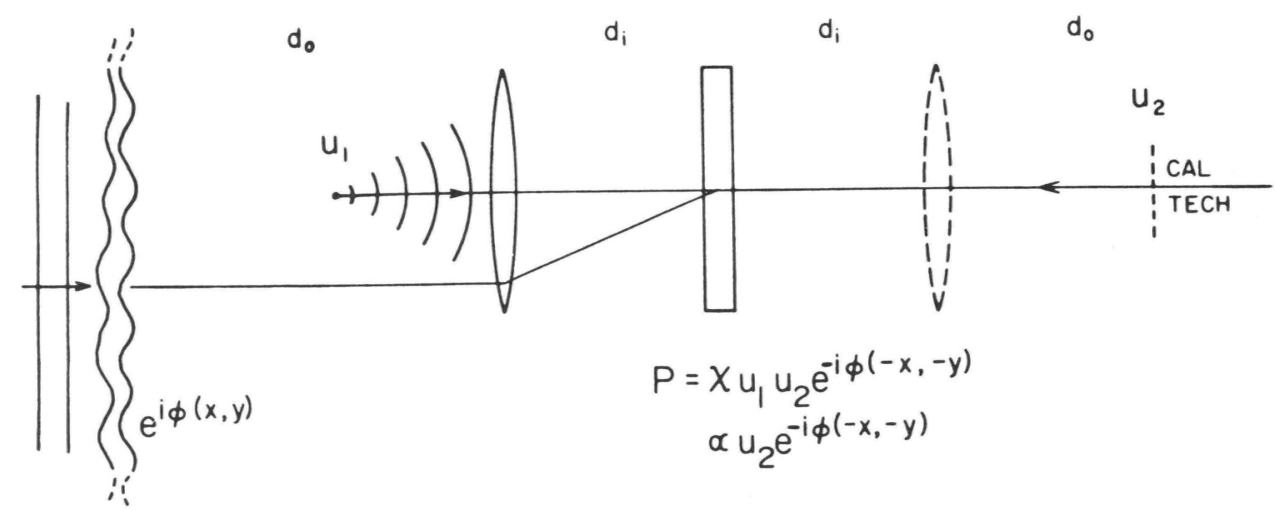

Figure 12. Technique for one-way field transmission through a thin phase distortion.

a)

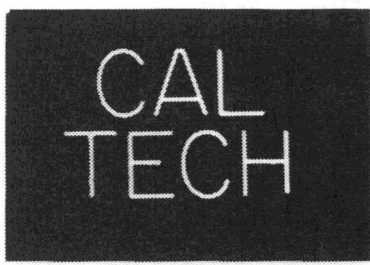

c)

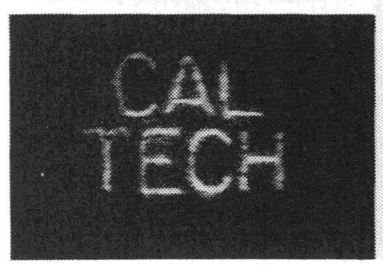

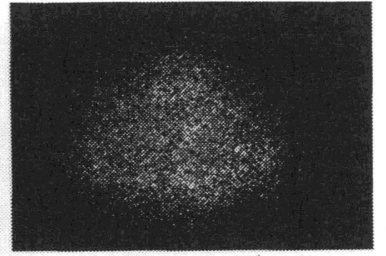

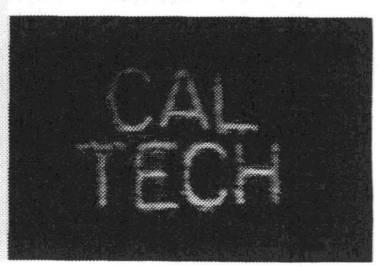

b)

d)

Figure 13. a) The object to be transmitted. b) A photograph of the object field transmitted through the distortion. c) The field transmitted through the phase conjugate window. d) The field transmitted through the crystal alone.

The conjugate distortion is created by nonlinear mixing of a distorted and an undistorted reference wave. The undistorted reference originates as a plane wave on the same side of the distortion as the object. The distorted reference, or beacon, originates on the opposite side of the distortion, and propagates through it. Both references are incident upon the nonlinear medium and a phase hologram is formed which is a conjugate replica of the distortion. The object field $\mathrm{A}_{2}$ is then able to pass unaberrated through the hologram-lens-distortion combination, which is called a phase conjugate window. Intensity information is commonly transmitted through a thin phase distortion, without the need of a nonlinear medium, by imaging the object onto the distortion, e.g., with the dashed lens in Fig. 12. Our nonlinear optical technique transmits both the amplitude and the phase of the object field, without the second lens.

The object used in the experiment is a transparency shown in Fig. 13a. The distortion is a piece of ground glass. The object and distortion are both illuminated in transmission. All three waves incident upon the crystal $\left(\mathrm{BaTiO}_{3}\right)$ are provided by the same argon ion laser operating at $514.5 \mathrm{~nm}$. The object wavefront is obliterated by the random phase distortion (Fig. 13b). Fig. $13 \mathrm{c}$ is an image of the object field transmitted through the phase conjugate window, showing almost ccmplete reconstruction. Fig. 13d is an image of the object field transmitted through the crystal alone, showing that the residual aberrations in $13 \mathrm{c}$ are due to inhomogeneities within the crystal itself. 
A point source or a properly oriented point reflector can also be used as a beacon in another scheme which also employs both a distorted and undistorted reference [54,55]. The Fourier transform of the object is incident on the nonlinear medium, which is BSO. For distortions which are weak and located close to the nonlinear medium, it may not be necessary to image the two together.

\section{Through a multi-mode fiber}

Imaging a three-dimensional object through a multi-mode fiber is difficult because of modal distortion, differential modal attenuation, and intermode scattering. The first problem can be solved using optical phase conjugation [56] and has been demonstrated using D4WM [57]. A picture field at the entrance to a fiber excites many eigenmodes, which propagate with different phase velocities. The phase differences which build up, after propagating through half the length of the fiber, can be negated with a phase conjugate mirror, and then cancelled upon propagating the field through the second half of the fiber. The experiment was performed with $\mathrm{BaTiO}_{3}$, and a forward and backward pass through the same piece of fiber.

\section{Spatial Information Processing}

Coherent and incoherent optical processors have been used for pattern recognition, guidance systems, image deblurring, edge enhancement, and synthetic aperturing. Van der Lugt filters have typically been made from photographic film, which requires a developing stage. A nonlinear medium can be substituted for the film, allowing for rapid creation and modification of the filter.

Van der Lugt filtering

The utility of gratings for optical processing has been demonstrated [58]. For example, a grating placed in the filter plane of a coherent optical processor can translate, add, and subtract input pattern functions. Multiple gratings can also be used to obtain first- or higher-order derivatives of intensity distributions, and restore smeared or multiply exposed images.

The concept can be generalized beyond gratings in the filter plane to the production of more complicated Van der Lugt filters [59]. In the Van der Lugt technique, a filter or mask is first synthesized by exposing film to the interference pattern of a plane reference wave and the Fourier transform, $\mathrm{H}$, of the desired impulse response, $\mathrm{h}$. $\mathrm{H}$ is obtained in the back focal plane of a lens when $h$ is in the front focal plane. The developed film is then reinserted into the filter plane, and the object $u$ to be processed is inserted into the input plane in place of $h$. Now the field $U$ is incident upon the filter and one component of the field leaving the transform plane is proportional to the product HU. Propagating through the second lens gives $h * u$ at the output plane, by the convolution theorem, where $*$ denotes convolution.

Some caution must be exercised when substituting a nonlinear medium for the film. For real-time operation of the processor, $h$ and $u$ must be present concurrently, and if one is off-axis, astigmatism can result [60]. Strictly speaking, for an object in the front focal plane of a lens, the exact Fourier transform only exists at one plane in space, the back focal plane. Depending on the confocal parameter of the system, a good or bad approximation to the transform may exist in some noninfinitesimal region about the focal plane [59]. This puts a limit on the thickness, $t$, of both volume filters and film emulsions, $t<2 f^{2} \lambda / r_{\max }^{2}$, where $r_{\max }$ is the spatial extent of the largest input field, and $f$ is the focal length of the lens.

Also, filters made from photographic film are unaffected by light after the developing process, whereas photorefractive materials remain sensitive to light in the absence of some fixing process [18]. Birefringent and optically active nonlinear media introduce more complications that can turn out to be useful for improving performance [61]. Finally, we know that volume gratings have amplitude transmittances that depend strongly on the wavelength and angle of illumination [29]. So, the synthesis of a dynamic volume filter must be done keeping the intensity, direction, wavelength, and polarization of all beams in mind.

Spatial convolution and correlation

The multiplicative properties of nonlinear optics can be combined with the Fourier transforming properties of lenses to yield spatial convolution and correlation. In a variation on the Van der Lugt sequence, the transformed filter function and input field interfere to write a hologram, which is then read out with a uniform plane wave [62]. The input objects are illuminated with the $488 \mathrm{~nm}$ wavelength from an argon laser, and a $632.8 \mathrm{~nm}$ HeNe beam is used for the reference beam, which is almost superimposed with one of the inputs, but misaligned slightly so as to be incident at the Bragg angle. The photorefractive material is BSO, which is less sensitive to red than blue, so the reference beam provides a nearly nondestructive readout.

A solution which requires only one wavelength is D4WM. In the grating picture, it corresponds to illumination at the Bragg angle, but in the reverse direction. Again the nonlinear medium is placed in the common focal plane of a two lens system as shown in Fig. 14. The three input fields, located in the outer focal planes, contain arbitrary amplitude and phase information. The input complex amplitudes $u_{1}(x, y), u_{2}(x, y)$, and $u_{4}(x, y)$, are Fourier transformed by propagating to the common focal plane. In the nonlinear optics picture, the transformed fields $U_{1}, U_{2}$, and $U_{4}$ induce nonlinear polarizations in the medium. The polarization $P=\chi^{(3)} U_{1} U_{2} U_{4}^{*}$ radiates an output field that propagates essentially backward relative to beam four, returning through lens $L$. The convolution theorem says that the transform of the product $U_{1} U_{2}$ is the convolution of the transforms $u_{1} * u_{2}$. Accordingly, it can be shown that the output field, when evaluated at the plane located a distance $f$ in front of lens $L$, is of the form $u_{3} \propto u_{1} * u_{2} \otimes u_{4}$, where $\otimes$ denotes correlation [63]. 
This operation was demonstrated with the experimental arrangement shown in Fig. 14, where the nonlinear medium is BSO [64]. The interaction is exactly phase matched only when $U_{1}$ and $U_{2}$ are counterpropagating. This puts a constraint on the spatial extent of the input fields which can be shown to be the same as the above constraint on filter thickness. Typical

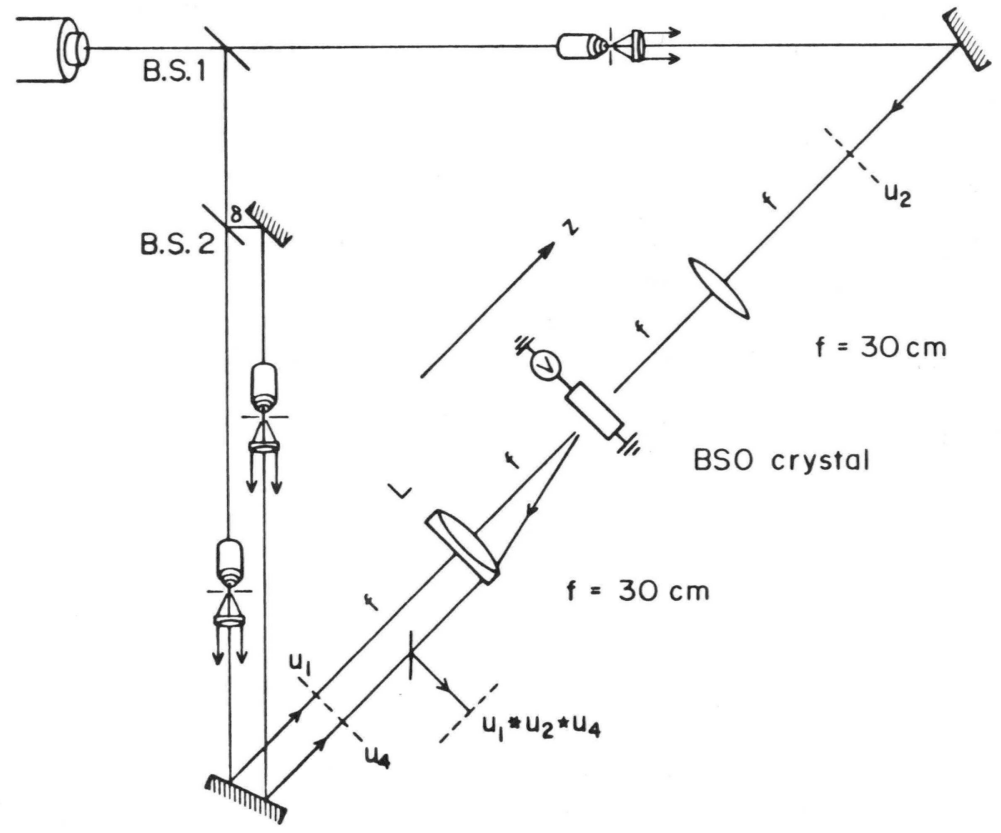

Figure 14. Four-wave mixing configuration for performing spatial convolution and correlation of images.

a)

\begin{tabular}{|c|c|c|c|}
\hline$U_{1}$ & $U_{2}$ & $U_{4}$ & $U_{3}$ \\
\hline$\bullet$ & DELTA & $\bullet \cdot$ & $* * * *$ \\
\hline & FUNCTION & $\bullet \cdot$ & $* *$ \\
\hline
\end{tabular}

b)

c)

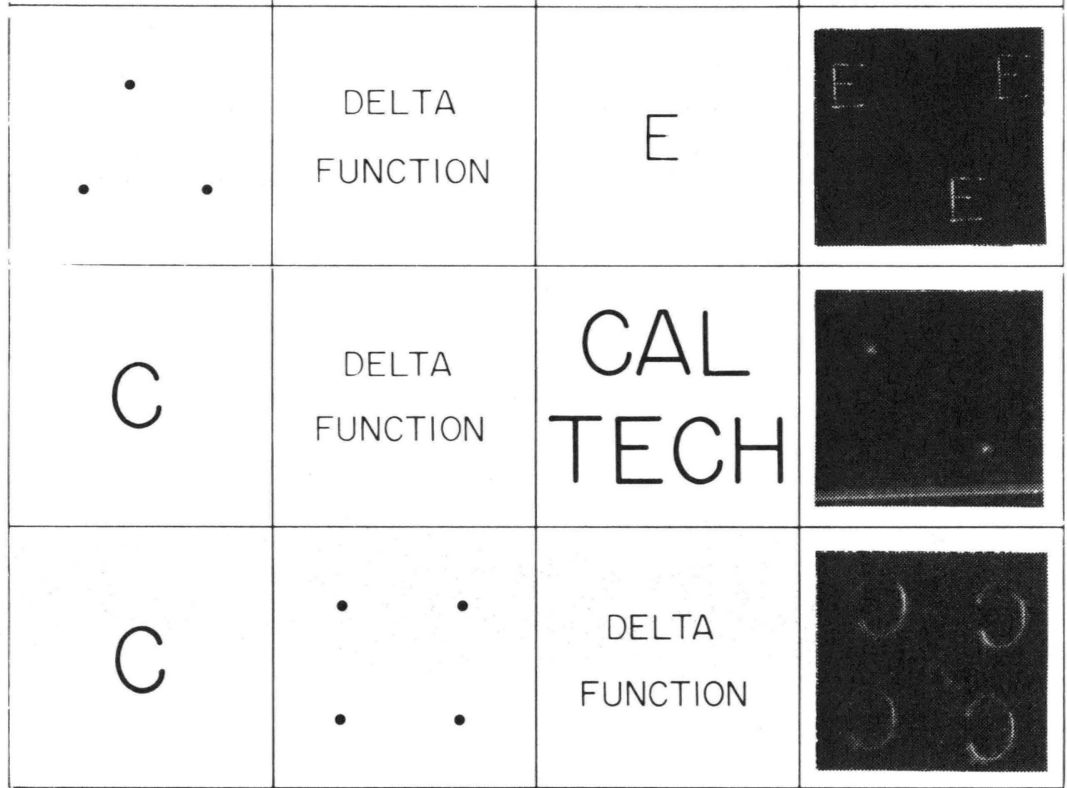

Figure 15. Output of the processor shown in Fig. 14. 
experimental results are shown in Fig. 15. The first three columns show the input fields $u_{1}, u_{2}$, and $u_{4}$. The fourth column shows the photograph at the output plane. Rows (a) to (c) illustrate correlation, and row (d) illustrates convolution. The output in row (c) clearly demonstrates pattern recognition.

Some deviation from perfect convolution must be expected because the photorefractive nonlinearity is not simply described by a multiplication of field amplitudes; the product is normalized by the average intensity. The next section describes how the photorefractive nonlinearity can be used in edge enhancement.

\section{Edge enhancement}

The special type of nonlinearity present in the photorefractive effect can be used to advantage in image division [65] and edge enhancement. Since the amplitude of the index modulation is proportional to the local fringe modulation, an increase in the intensity of one of the optical fields will not necessarily yield a larger effect. The largest index modulation (most efficient hologram) will be produced when two optical fields of the same intensity interfere. So, if one wants to enhance the boundary of an object field containing a very intense region and a dark region, choose a reference beam with an intermediate intensity, $I^{\prime}$. Image the object onto a photorefractive crystal along with the reference. The largest interference fringe modulation will occur at the boundary between the light and dark regions of the image (Figure 16).

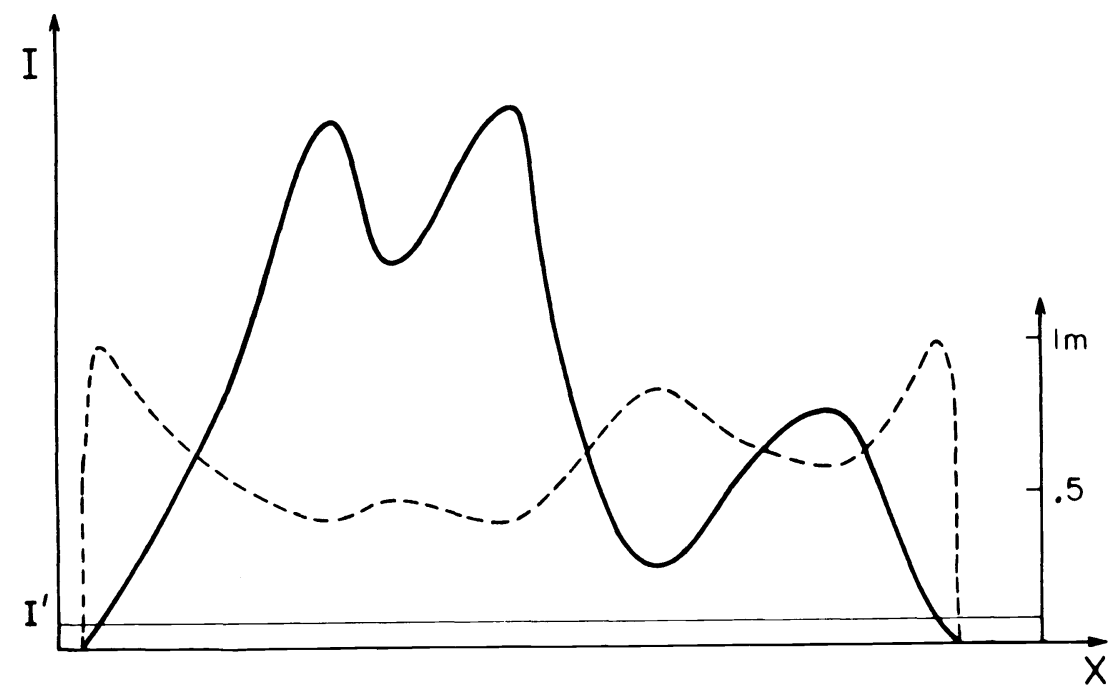

Figure 16. The mechanism of edge enhancement using the photorefractive effect.

When the hologram is illuminated with a weak, third, planar wavefront, the regions of the crystal with the largest index modulation will diffract the most light, yielding an intensity filtering in the image plane [66]. A different type of filtering can also be done by performing the operation in the transform plane. Horizontal or vertical edges can be selectively enhanced by a slight misalignment of the beams [67]. Reflection holograms can be used [68] as well as transmission holograms.

\section{Acknowledgements}

The authors gratefully acknowledge the support of the U.S. Air Force Office of Scientific Research, and the U.S. Army Research Office, Durham, N. Carolina.

\section{References}

1. A. Ashkin, G.D. Boyd, J.M. Dziedzic, R.G. Smith, A.A. Ballman, J.J. Levinstein \& K. Nassau, Appl. Phys. Lett. 9, 72 (1966).

2. J.L. Jackel, D.H. Olsen \& A.M. Glass, J. Appl. Phys. 52, 4855 (1981).

3. Yu.L. Kopylov, V.B. Kravchenko \& V.V. Kucha, Sov. Tech. Phys. Lett. 8, 88 (1982).

4. P. Gunter \& F. Micheron, Ferroelectrics 18, 27 (1978).

5. K.L. Sweeney \& L.E. Halliburton, Appl. Phys. Lett. 43, 336 (1983).

6. R. Courths, P. Steiner, H. Hochst \& S. Hufner, Appl. Phys. Lett. 21, 345 (1980). 
7. V.M. Bermudez, J.W. Hudgens \& M.A. Hoffbauer, Appl. Opt. 22, 3681 (19).

8. H. Kurz, E. Kratzig, W. Keune, H. Engelmann, U. Gonser, B. Dischler \& A. Rauber, Appl. Phys. 12, 355 (1977).

9. F.S. Chen, J. Appl. Phys. 38, 3418 (1967).

10. J.J. Amodei, RCA Review, 32, 185 (1971).

11. A.M. Glass, Opt. Engin. 17, 470 (1978).

12. N.V. Kukhtarev, V.B. Markov, S.G. Odulov, M.S. Soskin \& V.L.Vinetskii, Ferroelectrics 22, 949 (1979).

13. J. Feinberg, D. Heiman, A.R. Tanguay, Jr. \& R.W. Hellwarth, J. Appl. Phys. 51, 1297 (1980).

14. J.P. Huignard, J.P. Herriau, P. Auborg \& E. Spitz, Opt. Lett. 4, 21 (1979).

15. J.P. Huignard \& F. Micheron, Appl. Phys. Lett. 29, 591 (1976).

16. R. Grousson \& S. Mallick, Appl. Opt. 19, 1762 (1980).

17. F.S. Chen, J.T. LaMacchia \& D.B. Fraser, Appl. Phys. Lett. 13, 223 (1968).

18. D.L. Staebler, W.J. Burke, W. Phillips \& J.J. Amodei, Appl. Phys. Lett. 26, 182 (1975).

19. E.S. Vartanyan, E.Kh. Gulanyan \& R.K. Ovsepyan Sov. J. Quant. Elect. 10, 247 (1980).

20. J.E. Weaver \& T.K. Gaylord, Opt. Engin. 20, 404 (1981).

21. T.A. Belozerova, A.A. Komlev, N.F. Kovtonyuk, S.S. Kuz'min \& A.L. Mikaelyan, Sov. J. Quant. Elect. 12 , 1368 (1982).

22. M. Miteva, Opt. Comm. 48, 85 (1983).

23. J.P. Huignard, J.P. Herriau \& T. Valentin, Appl. Opt. 16, 2796 (1977).

24. J.P. Huignard \& J.P. Herriau, Appl. Opt. 16, 1807 (1977).

25. P. Auborg, J.P. Huignard, M. Hareng \& R.A. Mullen, Appl. Opt. 21, 3706 (1982).

26. Y. Shi, D. Psaltis, A. Marrakchi \& A.P. Tanguay Jr., Appl. Opt. 22, 3665 (1983). See also papers $465-01$ and $465-12$ in this issue.

27. A.A. Kamshilin \& M.P. Petrov, Sov. Tech. Phys. Lett. 6, 144 (1980).

28. D.L. Staebler \& J.J. Amodei, J. Appl. Phys. 43, 1042 (1972).

29. H. Kogelnik, Bell Syst. Tech. J. 48, 2909 (1969).

30. D.W. Vahey, J. Appl. Phys. 46, 3510 (1975).

31. F. Laeri, T. Tschudi \& J. Albers, Opt. Comm. 47, 387 (1983).

32. N. Kukhtarev, V. Markov \& S. Odulov, Opt. Comm. 23, 338 (1977).

33. J.O. White, M. Cronin-Golomb, B. Fischer \& A. Yariv, Appl. Phys. Lett. 40, 450 (1982).

34. D.M. Pepper, Ph.D. thesis, California Institute of Technology, 1980.

35. D.L. Fried, guest editor, Special Issue on Adaptive Optics, J. Opt. Soc. of Am. 67, No.3 (1977).

36. H. Kogelnik, Bell Syst. Tech. J. 44, 2451 (1965).

37. B.Y. Zeldovich, V.I. Popovichev, V.V. Ragulskii \& F.S. Faisulov, JETP Lett., 15, 109 (1972).

38. V. Wang \& C.R. Giuliano, Opt. Lett. 2, 4 (1978).

39. A. Yariv, IEEE J. Quant. Elect. 14, 650 (1978).

40. D.M. Pepper, guest editor, Special Issue on Optical Phase Conjugation, Opt. Engin. 21, No.2 (1982).

41. R.A. Fisher, editor, Optical Phase Conjugation, Academic Press, New York, 1983.

42. A. Yariv \& D.M. Pepper, Opt. Lett. 1, 16 (1977).

43. A. Yariv, Opt. Comm. 25, 23 (1978).

44. B. Fischer, M. Cronin-Golomb, J.O. White \& A. Yariv, Opt. Lett. 6, 519 (1981).

45. J.F. Lam, Appl. Phys. Lett. 42, 155 (1983). 
46. M. Cronin-Golomb, B. Fischer, J.O. White \& A. Yariv, Appl. Phys. Lett. 41, 689 (1982).

47. M. Cronin-Golomb, B. Fischer, J. Nilsen, J.O. White \& A. Yariv, Appl. Phys. Lett. 41, 219 (1982).

48. J. Feinberg, Opt. Lett. 7, 486 (1982).

49. R.A. McFarlane \& D.G. Steel, Opt. Lett. 8, 208 (1983).

50. A. Litvinenko \& S. Odoulov, Opt. Lett. 9, 68 (1984).

51. J.W. Goodman, W.H. Huntley Jr., D.W. Jackson \& M. Lehman, Appl. Phys. Lett. 8, 311 (1966).

52. A. Yariv \& T.L. Koch, Opt. Lett. 7, 113 (1982).

53. B. Fischer, M. Cronin-Golomb, J.O. White \& A. Yariv, Appl. Phys. Lett. 41, 141 (1982).

54. O. Ikeda, T. Suzuki \& T. Sato, Appl. Opt. 22, 2192 (1983).

55. O. Ikeda, T. Sato \& M. Takehara, Appl. Opt. 22, 3562 (1983).

56. A. Yariv, Appl. Phys. Lett. 28, 88 (1976).

57. G.J. Dunning \& R.C. Lind, Opt. Lett. 7, 558 (1972).

58. S.H. Lee, Opt. Engin. 13, 196 (1974).

59. J.W. Goodman, Introduction to Fourier Optics, McGraw-Hill, New York, 1968.

60. B. Carquille \& C. Froehy, Appl. Opt. 19, 207 (1980).

61. J.P. Herriau, J.P. Huignard \& P. Auborg, Appl. Opt. 17, 185 (1978).

62. L. Pichon \& J.P. Huignard, Opt. Comm. 36, 277 (1981).

63. D.M. Pepper, J. AuYeung, D. Fekete \& A. Yariv, Opt. Lett. 3, 7 (1978).

64. J.O. White \& A. Yariv, Appl. Phys. Lett. 37, 5 (1980).

65. Y.H. Ja, Opt. Comm. 44, 24 (1982).

66. J.P. Huignard \& J.P. Herriau, Appl. Opt. 17, 2671 (1978).

67. J. Feinberg, Opt. Lett. 5, 330 (1980).

68. Y.H. Ja, Opt. Quant. Elect. 15, 269 (1983). 